\title{
Nasal carriage of Staphylococcus aureus treated with topical mupirocin (pseudomonic acid) in a children's hospital
}

\author{
U. Frank, ${ }^{*}$ W. Lenz, $\dagger$ E. Damrath, ${ }^{*}$ I. Kappstein, ${ }^{*}$ \\ and F. D. Daschner* \\ *Department of Hospital Epidemiology, University Hospital, Freiburg and \\ $\uparrow$ National Reference Centre for Staphylococcal Typing, Institute for Medical \\ Microbiology, Bonn, FRG
}

Accepted for publication 24 May 1988

\begin{abstract}
Summary: $2 \%$ mupirocin ointment applied intra-nasally for 5 days was assessed for elimination of nasal carriage of Staphylococcus aureus in 31 staff members in a children's hospital. Three volunteers failed to complete the trial because of side effects, i.e. buccal reddening and swelling, and unpleasant taste. During treatment staphylococcal nasal carriage was not found in any case; of the 24 post-treatment nasal swabs taken 4 days after treatment 22 were still negative. Re-colonization with $S$. aureus of different phage types occurred in the remaining two cases.
\end{abstract}

Key words: Nasal staphylococcal carriage; topical mupirocin; pseudomonic acid.

\section{Introduction}

Fuller et al. (1971) first recorded the antibacterial activity of a group of substances produced by fermentation of a strain of Pseudomonas fluorescens. Subsequently, the name pseudomonic acid $A$ was ascribed to the major metabolite, which accounted for most of the antibacterial activity observed. Mupirocin has been accepted as the approved name for pseudomonic acid A. Its chemical structure and mode of action has been shown to be different from any known class of antibiotics (Chain 8 Mellows, 1977; Hughes \& Mellows $1978 a, b$; Alexander et al., 1985).

Mupirocin has a narrow spectrum of activity, confined mainly to gram-positive bacteria, including Staphylococcus aureus, Staphylococcus epidermidis, and Streptococcus pyogenes. There is moderate activity against

Correspondence to: F. D. Daschner, Department of Hospital Epidemiology, University of Freiburg, Hugstetterstr. 55, 7800 Freiburg, FRG. 
some gram-negative organisms, such as Haemophilus influenzae and Neisseria gonorrhoeae, but it is relatively inactive against enterobacteria and enterococci (Sutherland et al., 1985). Casewell \& Hill (1985, 1986) demonstrated the good in-vitro activity of mupirocin against $S$. aureus, including methicillin-resistant strains and suggested mupirocin as the topical agent of choice to eliminate nasal carriage. Bactericidal concentrations of mupirocin are achieved by topical administration of a $2 \%$ formulation in an ointment. This study was carried out to assess the eflicacy of mupirocin in the elimination of nasal carriage of $S$. aureus in healthy volunteers.

\section{Materials and methods}

One hundred and eighty-six medical and nursing staff working in clinical areas of a children's hospital were screened for nasal and pharyngeal carriage of S. aureus. The organism was isolated from $63(33.9 \%)$, and 31 of these staff (nine males and 22 females), who were still positive after a repeat nasal swab at 1 week, were enrolled in the study. Informed consent was obtained from each subject.

The topical treatment consisted of pseudomonic acid A $2 \% \mathrm{w} / \mathrm{w}$ as calcium salt in an ointment base of soft paraffin containing anhydrous lanolin (Beecham Pharmaceuticals, Worthing, England). Volunteers were given verbal and written instructions. The ointment was applied in match-head size amounts to each nostril by fingertips. After application it was distributed by squeezing the nose between finger and thumb. The ointment was given four times a day for 5 days.

The persistence of staphylococcal carriage prior to treatment was confirmed by examination of a third nasal and pharyngeal swab. On the third day of treatment another swab was taken. Follow-up nasal swabs were collected 1 and 4 days after each course.

All nasal swabs were collected in a standard way by firmly rotating plain cotton-wool swabs, soaked in sterilized physiologic saline, around the periphery of the anterior nares. All specimens were inoculated on blood-agar and on mannitol salt agar plates (Columbia-Blutagar, Mannit-Kochsalz-Phenolrot-Agar, Heipha, Heidelberg, FRG) and incubated at $37^{\circ} \mathrm{C}$ for 24 and $48 \mathrm{~h}$. Colonies were identified as $S$. aureus if they showed typical morphology on blood agar, fermentation of mannitol salt agar, were gram-positive cocci in clusters and gave a positive reaction of the coagulase-slide or coagulase-tube test. Growth of $S$. aureus was read semi-quantitatively, i.e. a scanty, moderate, or heavy growth.

Phage typing of $S$. aureus was carried out by Dr. W. Lenz (National Reference Center for Staphylococcal Typing, Institute for Medical Microbiology, Bonn, Federal Republic of Germany). 


\section{Results}

Before treatment, all the 31 nasal swabs and $10(32 \cdot 3 \%)$ of pharyngeal swabs were positive for $S$. aureus. Three volunteers failed to complete the trial; one noticed buccal reddening and swelling and wished to stop and two found the ointment unpleasant to use. During treatment staphylococcal carriage could not be demonstrated in any volunteer; two swab results could not be obtained. Follow-up swabs 1 day after treatment failed to yield $S$. aureus with one exception or again in two cases swab results were not available. Of the 24 post-treatment swabs taken 4 days after the course was completed, $22(91.7 \%)$ were still negative for $S$. aureus, whereas recolonization of the anterior nares with $S$. aureus of different phage types was found in two volunteers.

\section{Discussion}

In the present study, mupirocin has been shown promptly to eliminate nasal carriage of $S$. aureus. As suggested in previous studies (Dacre, Emmerson \& Jenner, 1983; Casewell \& Hill, 1986), this topical agent has an important role in the elimination of nasal staphylococci. During a small outbreak of gentamicin-and methicillin-resistant $S$. aureus on a urology ward, mupirocin was found to be effective in cradicating the multiresistant micro-organism in 15 nasal carriers (Dacre, et al., 1983). However, the formulation in polyethylene glycol was associated with side effects such as localized stinging, soreness, dry skin and itching around the nose, noticed by six of the subjects.

In Casewell \& Hill's study (1986) of 32 healthy volunteers, no significant side-effects were observed with a white soft paraffin base formulation. In their study $S$. aureus could not be detected, even in low numbers, in nose swabs from any of the volunteers within 2 days of starting mupirocin, and even after 5 weeks only six showed recolonization. Ultimately, 14 individuals resumed staphylococcal carriage, in most cases with different phage types.

Similarly encouraging results were found in our study. Elimination of $S$. aureus from the anterior nares could be found on day 3 of therapy in all subjects from whom swabs were available. One week after starting the treatment recolonization was noted in only two subjects. Unfortunately, further follow-up could not be carried out. However, the prompt eradication of nasal carriage by mupirocin within 3 days of treatment was impressive, and gave further evidence that it may be useful in controlling staphylococcal outbreaks in hospitals.

\section{References}

Alexander, R. G., Clayton, J. P., Luk, K., Rogers, N. H. \& King, T. J. (1978). The chemistry of pseudomonic acid. Part 1 . The absolute configuration of pseudomonic acid A. Yournal of the Chemical Society Perkin I, 561-565.

Casewell, M. W. \& Hill, R. L. R. (1985). In-vitro activity of mupirocin (pseudomonic acid) 
against clinical isolates of Staphylococcus aureus. Fournal of Antimicrobial Chemotherapy $15,523-531$.

Casewell, M. W. \& Hill, R. L. R. (1986). Elimination of nasal carriage of Staphylococcus aureus with mupirocin (pseudomonic acid) - a controlled trial. Fournal of Antimicrobial Chemotherapy 17, 365-372.

Chain, E. B. \& Mellows, G. (1977). The structure of pseudomonic acid A, a novel antibiotic produced by Pseudomonas fluorescens. Journal of the Chemical Society Perkin I, 294-309.

Dacre, J. E., Emmerson, A. M. \& Jenner, E. A. (1983). Nasal carriage of gentamicin and methicillin resistant Staphylococcus aureus treated with topical pseudomonic acid. Lancet ii, 1036.

Fuller, A. T., Mellows, G., Woolford, G. T., Banks, G. T., Barrow, K. D. \& Chain, E. B. (1971). Pseudomonic acid: an antibiotic produced by Pseudomonas fuorescens. Nature 234, 416-417.

Hughes, J. \& Mellows, G. (1978a). On the mode of action of pseudomonic acid: Inhibition of protein synthesis in Staphylococcus aureus. Fournal of Antibiotics 31, 330-335.

Hughes, J. \& Mellows, G. (1978b). Inhibition of isoleucyl-transfer ribonucleic acid synthetase in Escherichia coli by pseudomonic acid. Biochemistry fournal 191, 209-219.

Sutherland, R., Boon, R. J., Griffin, K. E., Masters, P. J., Slocombe, B. \& White, A. R. (1985). Antibacterial activity of mupirocin (pseudomonic acid), a new antibiotic for topical use. Antimicrobial Agents and Chemotherapy 27, 495-498. 